\title{
ORIGINAL RESEARCH \\ White Matter Involvement in Idiopathic Parkinson Disease: A Diffusion Tensor Imaging Study
}

\section{G. Gattellaro \\ L. Minati \\ M. Grisoli \\ C. Mariani \\ BACKGROUND AND PURPOSE: Diffusion tensor imaging (DTI) offers a unique window on the connec- tivity changes, extending beyond the basal ganglia, which accompany the cognitive symptoms of Parkinson disease (PD). The primary purpose of this study was to assess the microstructural damage to cerebral white matter occurring in idiopathic PD.}

F. Carella

M. Osio

E. Ciceri

A. Albanese

M.G. Bruzzone

MATERIALS AND METHODS: Our sample included patients with PD without dementia $(n=10$; Hoehn and Yahr stages I and II; Unified Parkinson Disease Rating Scale, $20.5 \pm$ 8.3; and Mini-Mental State Examination, $28.3 \pm 1.5)$ and age-matched healthy control subjects $(n=10)$. DTI was performed on a $1.5 T$ scanner, and mean diffusivity (MD) and fractional anisotropy (FA) maps were obtained. Regions of interest (ROls) were drawn on the major fiber bundles as well as on gray matter nuclei.

RESULTS: In patients, the MD was increased at borderline significance in the substantia nigra but was unaltered in the thalamus, globus pallidus, putamen, and in the head of the caudate nucleus. The FA and MD were unaltered in the corticospinal tract in the midbrain and at the level of the internal capsule, and in the splenium of the corpus callosum. By contrast, the MD was increased and the FA was decreased in the genu of the corpus callosum and in the superior longitudinal fasciculus; in the cingulum, only the MD was altered. The observed changes were not significantly lateralized.

CONCLUSIONS: Widespread microstructural damage to frontal and parietal white matter occurs already in the early stages of $\mathrm{PD}$.
S tructural MR imaging results are usually normal in paients with idiopathic Parkinson disease (PD), even in those with a long disease duration. The role of MR imaging is essentially confined to excluding secondary causes of the symptoms such as vascular lesions, and to confirming the absence of specific imaging features found in atypical parkinsonisms, such as the hot-cross sign in multiple system atrophy (MSA-C). ${ }^{1,2}$

Nuclear medicine techniques, namely single-photon emission CT and positron-emission tomography with use of specific tracers such as the DaTSCAN (GE Healthcare, Buckinghamshire, United Kingdom) currently provide the best diagnostic sensitivity and specificity, but their viability is limited by high cost and low availability of equipment. ${ }^{3}$

Several advanced MR imaging techniques have been considered as adjuncts to structural imaging. Quantification of iron deposits in the basal ganglia by means of $\mathrm{T} 2{ }^{*}$ relaxometry reveals significant differences between patients and control subjects, but large overlap between the 2 groups limits its diagnostic usefulness. ${ }^{4}$ More recently, the accumulation of pathologic changes in the substantia nigra was found to be detectable by means of inversion-recovery sequences, and partial differentiation between patients and control subjects was obtained in several studies. ${ }^{5,6}$ Diffusion-weighted imaging (DWI) and diffusion tensor imaging (DTI) provide quantita-

Received December 16, 2008; accepted after revision January 16, 2009

From the Neuroradiology Unit (G.G., L.M., M.G., E.C., M.G.B.), Science Direction Unit (L.M.), and 1st Neurology Department (F.C., A.A.), Fondazione IRCCS Istituto Nazionale Neurologico "Carlo Besta," Milan, Italy; and Neurology Department (G.G., C.M., M.O.), Ospedale Luigi Sacco, Milan, Italy.

This study was funded by the Fondazione IRCCS Istituto Neurologico "Carlo Besta."

Please address correspondence to L. Minati, Neuroradiology Unit, Fondazione IRCCS Istituto Nazionale Neurologico "Carlo Besta," Milan, Italy; e-mail: Iminati@istituto-besta.it

Indicates open access to non-subscribers at www.ajnr.org

DOI 10.3174/ajnr.A1556 tive measures of the microstructural integrity of nuclei and fiber tracts. It has been shown that the mean diffusivity (MD, also referred to as apparent diffusion coefficient, ADC), representative of the integrity of the cellular matrix, is significantly altered in the putamen in MSA-P and in progressive supranuclear palsy (PSP) with respect to patients with PD and healthy control subjects. ${ }^{7,8}$ Furthermore, it has been reported that $\mathrm{MD}$ values in the middle cerebellar peduncle differentiate MSA-P from other parkinsonian disorders. ${ }^{8}$ Changes in fractional anisotropy (FA) can reveal progressive degeneration of axons in the major fiber bundles. Recent studies found reduced FA in patients with PD along the nigrostriatal projections, in the frontal lobes, in premotor areas, and in the cingulum. ${ }^{9,10}$

Already in the early stages of the disease, patients show cognitive deficits that may manifest as mild cognitive impairment (MCI) with predominant executive dysfunction, with symptoms typical of frontal lobe damage as well as visuospatial deficits. $^{11-14}$

In our study, we aimed at corroborating and extending the existing literature pertaining to the application of DTI to idiopathic PD by using region-of-interest (ROI)-based measurements to characterize the connectivity changes that extend beyond the basal ganglia and that are hypothesized to parallel the cognitive symptoms of PD.

\section{Materials and Methods}

\section{Subjects}

Ten patients with idiopathic PD ( 5 men and 5 women; mean age, $63.8 \pm 15.7$ years) and 10 control subjects ( 5 men and 5 women, mean age, $58.1 \pm 8.0$ years) were enrolled. The 2 groups were matched for sex $(P=1)$ and age $(P=.2)$. All patients were studied while undergoing MR imaging as a part of their clinical work-up. Control subjects were recruited among medics and paramedics. The purpose of the study was explained to all patients and control subjects, who were 
unpaid, and written informed consent for imaging and research use of the data were obtained. The study complied with applicable guidelines and regulations and was conducted in accordance with the principles set forth in the Helsinki declaration.

All control subjects had a negative anamnesis for neurologic and psychiatric disorders, no relative with a diagnosis of parkinsonism, and no abnormalities on structural MR imaging (see next subsection). To exclude significant impairment of global cognitive functioning, all subjects were assessed with the Mini-Mental State Examination and had a total score greater than 24; there was no significant difference between patients and control subjects ( $28.3 \pm 1.5$ vs $29.3 \pm$ $1.1 ; P=.1) .{ }^{15}$ None of the patients met the Diagnostic and Statistical Manual of Mental Disorders, Fourth Edition, criteria for PD with dementia. On the Beck Depression Inventory, all subjects had a total score lower than 13 , indicating minimal or no depression; the scores were higher in patients compared with control subjects $(4.8 \pm 4.1$ vs $0.9 \pm 1.1 ; P=.007) .{ }^{16}$

The diagnosis of PD was established by means of the clinical criteria of Gelb. ${ }^{17}$ For all patients, a detailed clinical history was taken, and a full neurologic examination was performed to exclude the presence of symptoms of atypical parkinsonisms. As confirmed by a senior neuroradiologist, no patient had any MR imaging finding suggesting a diagnosis of atypical parkinsonism or a secondary cause of the symptoms. In particular, atrophy of the midbrain and diencephalon and T2-signal intensity abnormalities in the periaqueductal area, suggestive of PSP, were absent. There was no evidence of atrophy of the pons, middle cerebellar peduncles, cerebellum, or putamen or of T2-signal intensity abnormalities in the cerebellar white matter, transverse pontine fibers, or putamen suggestive of MSA. Atrophy and T2-signal intensity abnormalities in the parietal and frontal cortical regions, suggestive of corticobasal degeneration, were also absent, and the ventricular size was normal for all patients. ${ }^{2}$ Two initially enrolled patients were excluded from the study because of the presence of multiple bilateral hyperintensities, suggesting vascular encephalopathy. All patients were taking levodopa, ropinirole, or pramipexole, alone or in combination, with a satisfactory response. Clinical follow-up 18 months or more after MR imaging further confirmed the absence of symptoms of atypical parkinsonisms and PD dementia, and stable response to therapy.

All patients were in stages I or II on the Hoehn and Yahr Scale. ${ }^{18}$ We assessed the severity of symptoms using the Unified Rating Scale for Parkinsonism in an on-medication state (total score, $20.5 \pm 8.3$; motor score, $14.2 \pm 6.5) .{ }^{19}$ The disease duration, measured from the self-reported onset of the symptoms, was $3.4 \pm 2.9$ years.

\section{Image Acquisition}

We performed MR imaging using a 1.5T scanner (Magnetom Avanto; Siemens, Erlangen, Germany) equipped with a 4-channel receiveonly head coil. All subjects were imaged with an axial double-echo T2 and proton-attenuation-weighted sequence. Imaging parameters were TR, $3500 \mathrm{~ms}$; TE, 17 and $84 \mathrm{~ms}$; FOV, $230 \times 187 \mathrm{~mm}$; matrix, $256 \times 208$; thickness, $6.0 \mathrm{~mm}$; and intersection gap, $10 \%$. Subjects were also imaged with a coronal fluid-attenuated inversion recovery sequence, with TR, $9040 \mathrm{~ms}$; TI, $2500 \mathrm{~ms}$; TE, $116 \mathrm{~ms}$; FOV, $231 \times 150$ $\mathrm{mm}$; matrix, $256 \times 166$; thickness, $5.0 \mathrm{~mm}$; and intersection gap, $10 \%$.

We performed DTI using an axial spin-echo echo-planar imaging sequence, with TR, $4200 \mathrm{~ms}$; TE, $79 \mathrm{~ms}$; FOV, $180 \times 240 \mathrm{~mm}$; matrix, $96 \times 128$; thickness, $2.5 \mathrm{~mm}$; and no intersection gap. Measurements were performed on 12 noncollinear directions at $b=0$ and 1000 $\mathrm{s} / \mathrm{mm}^{2}$, with 10 repetitions divided in 5 runs. After determination of the elements of the diffusion tensor by means of the least-squares method, the MD and FA were computed with standard formulas.

\section{Data Analysis}

Average MD and FA were measured by means of planar, elliptical ROIs, positioned as shown in Fig 1. All ROIs were individually drawn for each subject and hemisphere by an experienced neuroradiologist strictly blinded to disease status, and independently checked by another neuroradiologist, who always confirmed adherence to the anatomic criteria specified below. The ImageJ program (National Institute of Mental Health, Bethesda, Md) was used for this purpose.

The following ROIs were used to measure the MD in gray matter nuclei: an ROI positioned on the expected extension of the substantia nigra at the level of the red nucleus (average area, $0.5 \mathrm{~cm}^{2}$ ), an ROI positioned on the thalamus next to the interthalamic adhesion at the level of the internal capsule $\left(0.7 \mathrm{~cm}^{2}\right)$, and 3 ROIs positioned on the globus pallidus $\left(0.5 \mathrm{~cm}^{2}\right)$, putamen $\left(0.7 \mathrm{~cm}^{2}\right)$, and head of the caudate nucleus $\left(0.4 \mathrm{~cm}^{2}\right)$ at the level of the internal capsule. The following ROIs were used to measure the MD and FA in the white matter regions: an ROI positioned on the corticospinal tract (assumed to correspond to the central third of the cerebral peduncle, average area, 0.2 $\mathrm{cm}^{2}$ ) at the level of the red nucleus, an ROI positioned on the corticospinal tract at the level of the internal capsule $\left(0.5 \mathrm{~cm}^{2}\right), 2$ ROIs positioned on the genu and splenium of the corpus callosum $(0.6$ $\mathrm{cm}^{2}$ ), an ROI positioned on the superior longitudinal fasciculus at the level of the body of the corpus callosum $\left(3 \mathrm{~cm}^{2}\right)$, and an ROI positioned on the cingulum above the body of the corpus callosum $(0.8$ $\mathrm{cm}^{2}$ ).

The gray matter nuclei were included because of their involvement in a range of extrapyramidal movement disorders and to exclude the remote effects of nigral degeneration. The corpus callosum, superior longitudinal fasciculus, and cingulum -3 major associative bundles-were included because we hypothesized that they would be involved in the degeneration processes leading to the cognitive symptoms of PD; by contrast, the corticospinal tract was included as an internal control, given that its involvement in $\mathrm{PD}$ is not expected. ${ }^{7-10}$ The ROI sizes were determined during preliminary analysis of the data, with the purpose of maximizing coverage of the structures of interest to reduce the sensitivity to local variations, while minimizing partial-volume effects with neighboring areas. For each ROI, a 2-way analysis of variance (ANOVA) was performed, including a factor for subject group (patient or control subject) and a factor for side (left or right); for the ROIs corresponding to the genu and splenium of the corpus callosum, only the factor for subject group was used. To remove the potentially confounding effect of imperfect age matching, we introduced age into the model as a covariate. We searched for main effects of subject group and interactions between subject group and side (which would indicate a lateralization of pathologic change). To exclude deviation from a normal distribution, Kolmogorov-Smirnov tests were performed for each ANOVA cell.

\section{Results}

All measurements were normally distributed (KolmogorovSmirnov $P>.1$ ). As reported in Table 1 , the MD was not altered in the thalamus, globus pallidus, putamen, or head of the caudate nucleus. However, there was a borderline effect toward increased MD in the substantia nigra $(7 \% ; P=.05)$.

As reported in Table 2, the corticospinal tract (both at the level of the cerebral peduncle and at the level of the internal 

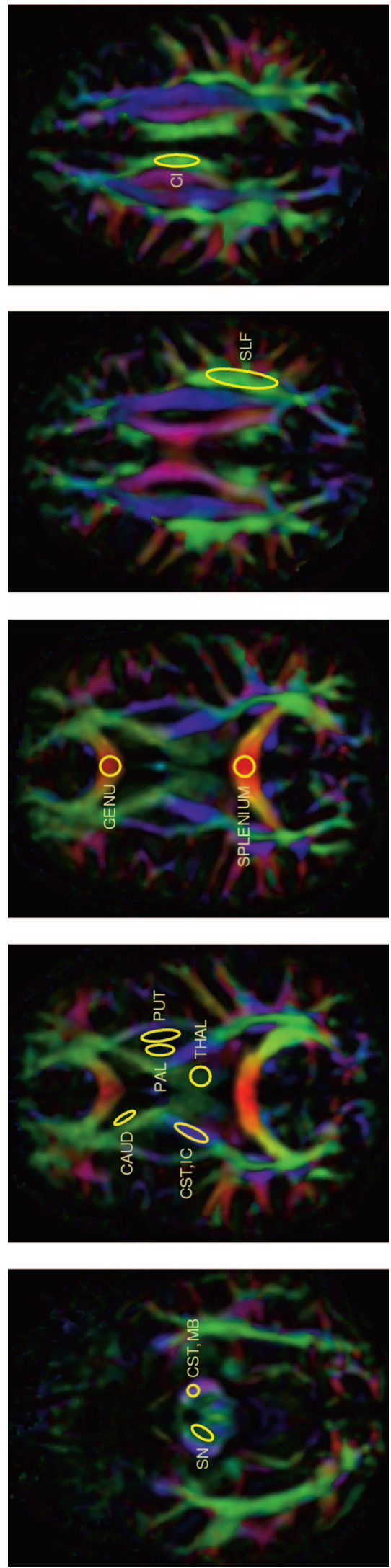

Fig 1. Positioning of the ROls used to measure $M D$ and $F A$, for an arbitrarily chosen subject. $S N$ : substantia nigra, CST,MB: corticospinal tract in the midbrain, CST,IC: corticospinal tract in the internal capsule, CAUD: head of the caudate nucleus, THAL: thalamus, PAL: globus pallidus, PUT: putamen, GENU: genu of the corpus callosum, SPLENIUM: splenium of the corpus callosum, SLF: superior longitudinal fasciculus, $\mathrm{Cl}$ : cingulum. capsule) and the splenium of the corpus callosum had unaltered MD and FA. By contrast, in patients the MD was higher and the FA was lower in the genu of the corpus callosum (19\%; $P=.002$, and $-14 \% ; P=.02$, respectively) and in the superior longitudinal fasciculus $(6 \% ; P=.01$, and $-8 \% ; P=.02$, respectively). In the cingulum, the MD was higher $(6 \% ; P=$ $.005)$, but the FA was unaltered. For all regions, the differences observed between patients and control subjects were not significantly different between the 2 hemispheres.

\section{Discussion}

Our results confirm those of previous studies showing that the thalamus, globus pallidus, putamen, and caudate nucleus are relatively spared in PD as opposed to atypical parkinsonisms. ${ }^{7,8}$ Increased MD in the substantia nigra may be consequential to the rarefaction of the cellular matrix, which follows extensive loss of dopaminergic neurons. ${ }^{20}$ Only 1 previous study included DTI measurements in the substantia nigra and, in line with our findings, reported decreased FA and a trend toward increased MD. ${ }^{21}$

Several neuroimaging studies have demonstrated cortical atrophy in patients with PD with and without dementia. Frontal lobe atrophy has been consistently found in patients with $\mathrm{PD}$ and without dementia, but reduced gray matter volume in the parietal and temporal regions seems to be more specifically associated with PD with dementia. ${ }^{22-25}$

Cognitive deficits indicating frontal lobe dysfunction typically accompany PD early in the course of the disease, and prefrontal atrophy has been found to correlate with attentional functioning in patients with early-stage PD. ${ }^{11-13,26}$ Functional MR imaging studies have demonstrated frontal dysfunction and reorganization manifesting, for example, as reduced functional connectivity during an attentional task, increased activation of premotor areas during a complex motor task, and reduced activation during a working memory task. $^{27-29}$ Increased MD and decreased FA in the genu of the corpus callosum indicate degeneration of the interhemispheric axonal connections between frontal areas and parallel the observed reduction in gray matter volume. ${ }^{22-25}$ This finding is in agreement with the results of a recent DTI study, in which voxel-based comparisons of FA measurements were performed. $^{10}$

The superior longitudinal fasciculus is composed of 4 bundles of axons, connecting multiple prefrontal and frontal regions with parietal and superior temporal areas. As a consequence of its structural heterogeneity, it is related to a range of premotor, motor, visuospatial, and auditory functions. ${ }^{30}$ Its involvement in PD probably parallels the observed deficits in premotor functions and visuospatial perception. ${ }^{11-13,26}$ Furthermore, a recent study has shown that reduced FA in the parietal white matter correlates with executive impairment in patients with PD without dementia. ${ }^{31}$ Increased MD and reduced FA in the superior longitudinal fasciculus are probably related to the degeneration of the temporal and parietal cortical regions, which becomes evident as loss of gray matter volume only in patients with dementia. ${ }^{22-25}$ In patients with no dementia, altered functionality of the parietal cortex has been reported in response to visual stimulation during attentional and complex motor tasks. ${ }^{27,28,32}$ To our knowledge, this study is the first to show that the superior longitudinal fasciculus, 
Table 1: Mean diffusivity (expressed as mean \pm SD) for the gray matter nuclei in patients and control subjects, and corresponding statistical findings

\begin{tabular}{llllr}
\hline & Control & PD & Control vs PD & Group x Side Interaction \\
\hline SN & $0.74 \pm 0.04$ & $0.79 \pm 0.08$ & $\mathrm{~F}=4.1 ; P=.05^{*}$ & $\mathrm{~F}=3.6 ; P=.08$ \\
PUT & $0.69 \pm 0.06$ & $0.71 \pm 0.04$ & $\mathrm{~F}=0.04 ; P=.8$ & $\mathrm{~F}=0.3 ; P=.6$ \\
PAL & $0.72 \pm 0.08$ & $\mathrm{~F}=0.9 ; P=.4$ & $\mathrm{~F}=0.02 ; P=.9$ \\
THAL & $0.73 \pm 0.06$ & $\mathrm{~F}=0.02 ; P=.9$ & $\mathrm{~F}=0.4 ; P=.5$ \\
CAUD & $0.66 \pm 0.16$ & $\mathrm{~F}=2.2 ; P=.1$ & $\mathrm{~F}=1.4 ; P=.2$ \\
\hline
\end{tabular}

Note:-SN indicates substantia nigra; PUT, putamen; PAL, globus pallidus; THAL, thalamus; CAUD, head of the caudate nucleus; PD, Parkinson disease.

* Indicates statistical significance. Diffusivity values are expressed as $\times 10^{-3} \mathrm{~mm}^{2} \mathrm{~s}^{-1}$

Table 2: Mean diffusivity (expressed as mean \pm SD) for the white matter regions in patients and control subjects, and corresponding statistical findings

\begin{tabular}{lcclc}
\hline & Control & PD & Control vs PD & Group x Side Interaction \\
\hline CST,MB & $0.69 \pm 0.05$ & $0.71 \pm 0.05$ & $\mathrm{~F}=2.8 ; P=.1$ & $\mathrm{~F}=2.7 ; P=.1$ \\
CST,PLIC & $0.69 \pm 0.05$ & $0.68 \pm 0.03$ & $\mathrm{~F}=2.5 ; P=.1$ & $\mathrm{~F}=0.05 ; P=.8$ \\
SLF & $0.68 \pm 0.04$ & $0.72 \pm 0.06$ & $\mathrm{~F}=7.3 ; P=.01^{*}$ & $\mathrm{~F}=0.4 ; P=.5$ \\
CI & $0.65 \pm 0.03$ & $0.69 \pm 0.05$ & $\mathrm{~F}=14 ; P=.005^{*}$ & $\mathrm{~F}=0.2 ; P=.6$ \\
GENU & $0.58 \pm 0.05$ & $\mathrm{~F}=1.5 ; P=.2$ & \\
SPLENIUM & $0.68 \pm 0.03$ & $0.70 \pm 0.05$ & \\
\hline
\end{tabular}

Note:-CST,MB indicates corticospinal tract in the midbrain; CST,PLIC, corticospinal tract at the level of the cerebral peduncle and internal capsule; SLF, superior longitudinal fasciculus; $\mathrm{Cl}$, cingulum; GENU, genu of the corpus callosum; SPLENIUM, splenium of the corpus callosum.

${ }^{*}$ Indicates statistical significance. Diffusivity values are expressed as $\times 10^{-3} \mathrm{~mm}^{2} \mathrm{~s}^{-1}$

\begin{tabular}{|c|c|c|c|c|}
\hline & Control & PD & Control vs PD & Group $x$ Side Interaction \\
\hline$\overline{C S T, M B}$ & $0.62 \pm 0.06$ & $0.61 \pm 0.06$ & $\mathrm{~F}=1.8 ; P=.2$ & $\mathrm{~F}=2.4 ; P=.1$ \\
\hline CST,PLIC & $0.51 \pm 0.04$ & $0.52 \pm 0.04$ & $\mathrm{~F}=0.01 ; P=.9$ & $\mathrm{~F}=1.0 ; P=.3$ \\
\hline SLF & $0.50 \pm 0.03$ & $0.46 \pm 0.05$ & $\mathrm{~F}=5.9 ; P=.02^{*}$ & $\mathrm{~F}=0 ; P=1$ \\
\hline $\mathrm{Cl}$ & $0.52 \pm 0.04$ & $0.50 \pm 0.04$ & $\mathrm{~F}=2.2 ; P=.2$ & $\mathrm{~F}=0.4 ; P=.5$ \\
\hline GENU & $0.74 \pm 0.05$ & $0.64 \pm 0.08$ & $\mathrm{~F}=6.8 ; P=.02^{*}$ & \\
\hline SPLENIUM & $0.73 \pm 0.06$ & $0.71 \pm 0.05$ & $\mathrm{~F}=0.4 ; P=.5$ & \\
\hline
\end{tabular}

* Indicates statistical significance.

known to degenerate in PSP, is also affected in patients with PD with no dementia. ${ }^{33}$

Although its exact functional correlates remain unclear, the cingulum subserves communication among different components of the limbic system and is involved in emotion formation, response initiation, and inhibition, as well as in learning. ${ }^{34}$ Atrophy in the limbic and paralimbic regions occurs in patients without dementia, though less markedly than in patients with dementia. ${ }^{24,25}$ Metabolic abnormalities in the posterior cingulate regions have also been reported. ${ }^{35}$ In our study, although the FA was not significantly reduced, the MD was significantly increased; in a previous study that considered FA only, alterations were found in the rostral and anterior cingulate regions. ${ }^{10}$

Diffusional parameters of the corticospinal tract were found to be unaltered, in agreement with a previous DTI study and with results from transcranial magnetic stimulation. ${ }^{36,37}$ Sparing of the splenium of the corpus callosum is also in agreement with a previous study. ${ }^{10}$

During the course of degenerative changes, in white matter the MD and FA are generally inversely correlated: as the relative attenuation of axonal membranes and myelin sheaths is reduced, diffusion becomes less anisotropic, and MD increases. ${ }^{38}$ In our study, the MD was elevated and the FA was reduced in the genu of the corpus callosum and in the superior longitudinal fasciculus; by contrast, in the cingulum the MD was elevated but the FA was not significantly altered. This dissociation is probably because the FA is much less homogeneous than the MD in the brain, and the larger variance potentially reduces the significance of group comparisons for some regions. ${ }^{38}$

Our study has limitations that need to be taken into account. Because no pathologic confirmation was available, a diagnosis of atypical parkinsonism could not be definitely excluded for our patients; however, the validity of the diagnosis was supported by the fact that, 18 months or longer after scanning, all of them remained free of symptoms of atypical parkinsonisms and continued responding satisfactorily to antiparkinsonian therapy. Detailed neuropsychological assessment was not performed. Therefore, though global cognitive functioning was not significantly impaired, the entity of the executive and visuospatial deficits in these patients was not known. The ROIs were drawn only once, and the reproducibility of the measurements was therefore not assessed; however, rater bias was prevented by blinding, and all ROIs were approved by a second neuroradiologist.

In conclusion, our study provided evidence for microstructural abnormalities extending beyond the basal ganglia in PD without dementia, occurring in the genu of the corpus callosum, in the superior longitudinal fasciculus, and in the cingulum. These changes were not significantly different between the 2 hemispheres. Our findings extend and largely confirm (except for the results obtained for the superior longitudinal fasciculus) those of a recent DTI study that was based on 
voxel-wise comparisons, an emerging technique known to deliver results potentially different from those obtained with ROI-based measurements. ${ }^{10,39}$ Despite being statistically significant, the differences observed in our study did not enable separation of patients and control subjects. This study only reported the presence of white matter abnormalities, without elucidating their functional correlates; additional studies are therefore needed, comparing patients in different stages of the disease and searching for correlations with specific neuropsychological tests.

\section{Acknowledgments}

We thank Dr. Tiziana De Simone for support during data acquisition, and 2 anonymous reviewers who provided insightful advice on an earlier draft.

\section{References}

1. Schrag A, Good CD, Miszkiel K. Differentiation of atypical parkinsonian syndromes with routine MRI. Neurology 2000;54:697-702

2. Savoiardo M, Grisoli M. Role of CT and MRI in diagnosis and research. In: Litvan I, ed. Atypical Parkinsonian Disorders: Clinical and Research Aspects. Totowa, NJ: Humana Press; 2005

3. Ravina B, Eidelberg D, Ahlskog JE, et al. The role of radiotracer imaging in Parkinson's disease. Neurology 2005;64:208-15

4. Vymazal J, Righini A, Canesi M, et al. T1 and T2 in the brain of healthy subjects, patients with Parkinson's disease, and patients with multiple system atrophy: relation to iron content. Radiology 1999;211:489-95

5. Hutchinson M, Raff U. Structural changes of tha substantia nigra in Parkinson's disease as revealed by MR imaging. AJNR Am J Neuroradiol 2000;21:697-701

6. Minati L, Grisoli M, Carella F, et al. Imaging degeneration of the substantia nigra in Parkinson's disease with inversion recovery MRI. AJNR Am J Neuroradiology 2007;28:309-13

7. Schocke MFH, Seppi K, Esterhammer R, et al. Trace of diffusion tensor differentiates the Parkinson variant of multiple system atrophy and Parkinson's disease. Neuroimage 2004;21:1443-51

8. Nicoletti G, Lodi R, Condino F, et al. Apparent diffusion coefficient measurements of the middle cerebellar peduncle differentiate the Parkinson variant of MSA from Parkinson's disease and progressive supranuclear palsy. Brain 2006;129:2679-87

9. Yoshikawa K, Nakata Y, Yamada K, et al. Early pathological changes in the parkinsonian brain demonstrated by diffusion tensor MRI. J Neurol Neurosurg Psychiatry 2004;75:481-84

10. Karagulle Kendi AT, Lehericy S, Luciana M, et al. Altered diffusion in the frontal lobe in Parkinson disease. AJNR Am J Neuroradiol 2008;29:501-05

11. Lees AJ, Smith E. Cognitive deficits in the early stages of Parkinson's disease. Brain 1983;106:257-70

12. Taylor AE, Saint-Cyr JA, Lang AE. Frontal lobe dysfunction in Parkinson's disease. The cortical focus of neostriatal outflow. Brain 1986;109:845-83

13. Farina E, Gattellaro G, Pomati S, et al. Researching a differential impairment of frontal functions and explicit memory in early Parkinson's disease. Eur J Neurol 2000;7:259-67

14. Janvin C, Larsen JP, Aarsland D, et al. Subtypes of mild cognitive impairment in Parkinson's disease: progression to dementia. Mov Disord 2006;21:1343-49

15. Folstein MF, Folstein SE, McHugh PR. Mini Mental State: a practical method for grading the cognitive state of patients for clinicians. J Psychiatry Res 1975;12:189-98
16. Beck AT, Steer RA, Brown GK. Manual for the Beck Depression Inventory-II. San Antonio, Tex: Psychological Corp; 1996

17. Gelb DJ, Oliver E, Gilman S. Diagnostic criteria for Parkinson's disease. Arch Neurol 1999;6:33-39

18. Hoehn MM, Yahr MD. Parkinsonism: onset, progression and mortality. Neurology 1967;17:427-42

19. Fahn S, Elton RL, members of the UPDRS Development Committee. Unified Parkinson's disease rating scale. In: Fahn S, Marsden CD, Goldstein M, et al, eds. Recent Developments in Parkinson's Disease Vol II. Florham Park, NJ: Macmillan Healthcare Information; 1987

20. Braak H, Del Tredici K, Rüb U, et al. Staging of brain pathology related to sporadic Parkinson's disease. Neurobiol Aging 2003;24:197-211

21. Chan LL, Rumpel H, Yap K, et al. Case control study of diffusion tensor imaging in Parkinson's disease. J Neurol Neurosurg Psychiatry 2007;78:1383-86

22. Double KL, Halliday GM, McRitchie DA, et al. Regional brain atrophy in idiopathic Parkinson's disease and diffuse Lewy body disease. Dementia 1996;7:304-13

23. Burton EJ, McKeith IG, Burn DJ, et al. Cerebral atrophy in Parkinson's disease with and without dementia: a comparison with Alzheimer's disease, dementia with Lewy bodies and controls. Brain 2004;127:791-800

24. Nagano-Saito A, Washimi Y, Arahata Y, et al. Cerebral atrophy and its relation to cognitive impairment in Parkinson disease. Neurology 2005;64:224-29

25. Beyer MK, Janvin CC, Larsen JP, et al. A magnetic resonance imaging study of patients with Parkinson's disease with mild cognitive impairment and dementia using voxel-based morphometry. J Neurol Neurosurg Psychiatry 2007;78:254-59

26. Brück A, Kurki T, Kaasinen V, et al. Hippocampal and prefrontal atrophy in patients with early non-demented Parkinson's disease is related to cognitive impairment. J Neurol Neurosurg Psychiatry 2004;75:1467-69

27. Sabatini U, Boulanouar K, Fabre N, et al. Cortical motor reorganization in akinetic patients with Parkinson's disease: a functional MRI study. Brain 2000;123:394-403

28. Rowe J, Stephan KE, Friston K, et al. Attention to action in Parkinson's disease: impaired effective connectivity among frontal cortical regions. Brain 2002;125:276-89

29. Lewis SJ, Dove A, Robbins TW, et al. Cognitive impairments in early Parkinson's disease are accompanied by reductions in activity in frontostriatal neural circuitry. J Neurosci 2003;23:6351-56

30. Makris N, Kennedy DN, McInerney S, et al. Segmentation of subcomponents within the superior longitudinal fascicle in humans: a quantitative, in vivo, DT-MRI study. Cereb Cortex 2005; 15:854-69

31. Matsui H, Nishinaka K, Oda M, et al. Wisconsin Card Sorting Test in Parkinson's disease: diffusion tensor imaging. Acta Neurol Scand 2007;1 16:108-12

32. Stebbins GT, Goetz CG, Carrillo MC, et al. Altered cortical visual processing in PD with hallucinations: an fMRI study. Neurology 2004;63:1409-16

33. Padovani A, Borroni B, Brambati SM, et al. Diffusion tensor imaging and voxel based morphometry study in early progressive supranuclear palsy. J Neurol Neurosurg Psychiatry 2006;77:457-63

34. Tekin S, Cummings JL. Frontal-subcortical neuronal circuits and clinical neuropsychiatry: an update. J Psychosom Res 2002;53:647-54

35. Camicioli RM, Korzan JR, Foster SL, et al. Posterior cingulate metabolic changes occur in Parkinson's disease patients without dementia. Neurosci Lett 2004;354:177-80

36. Eusebio A, Azulay JP, Witjas T, et al. Assessment of cortico-spinal tract impairment in multiple system atrophy using transcranial magnetic stimulation. Clin Neurophysiol 2007;118:815-23

37. Nilsson C, Markenroth Bloch K, Brockstedt S, et al. Tracking the neurodegeneration of parkinsonian disorders-a pilot study. Neuroradiology 2007; 49:111-19

38. Basser PJ, Pierpaoli C. Microstructural and physiological features of tissues elucidated by quantitative-diffusion-tensor MRI. J Magn Reson B 1996;111: 209-19

39. Snook L, Plewes C, Beaulieu C. Voxel based versus region of interest analysis in diffusion tensor imaging of neurodevelopment. Neuroimage 2007;34:243-52 